\title{
Retrospective analysis of 140 cases of medullary thyroid carcinoma followed-up in a single institution
}

\author{
JOANA SIMÕES-PEREIRA, MARIA JOÃO BUGALHO, EDWARD LIMBERT and VALERIANO LEITE \\ Department of Endocrinology, Portuguese Institute of Oncology Francisco Gentil, Lisbon 1099-023, Portugal
}

Received February 14, 2015; Accepted February 24, 2016

DOI: $10.3892 / \mathrm{ol} .2016 .4482$

\begin{abstract}
Familial cases of medullary thyroid carcinoma (MTC) may be diagnosed by genetic screening, while in sporadic tumors the diagnosis relies mainly on fine-needle aspiration cytology. The aim of the present study was to determine the demographic, clinical and pathological characteristics of MTC patients followed-up at the Portuguese Institute of Oncology Francisco Gentil (Lisbon, Portugal). For that purpose, a retrospective analysis of 140 MTC patients diagnosed between 1990 and 2010 was performed. The results indicated that patients with hereditary MTC (11.4\%) were significantly younger than patients with sporadic MTC. Of the latter, $34.3 \%$ had no clinical suspicion of MTC prior to surgery. The sensitivity of cytology and calcitonin (CT) assay in diagnosing MTC were 51.3 and $98.7 \%$, respectively. All familial index cases and $69.0 \%$ of sporadic cases presented with advanced stage disease at the time of diagnosis, while $73.0 \%$ of familial MTC detected by genetic/pentagastrin screening were diagnosed at the early stage of the disease. Biochemical cure (BC) was achieved in $39.7 \%$ of patients and, of these, only $6.5 \%$ relapsed. The 5 and 10 -year survival rates were 79.3 and $73.6 \%$, respectively. Age $>45$ years $(P=0.026)$, advanced stage at diagnosis $(\mathrm{P}<0.001)$ and absence of $\mathrm{BC}(\mathrm{P}<0.001)$ were predictors of a worse prognosis on univariate analysis. However, when the patients detected by genetic/pentagastrin screening were excluded from the analysis, age was no longer a prognostic factor, although disease stage remained a significant prognostic factor. On multivariate analysis, $\mathrm{BC}$ was the only factor with a significant impact on prognosis $(\mathrm{P}=0.031)$. In addition, the present study confirmed that the majority of patients were diagnosed at advanced stages, and CT determination was observed to be more sensitive than cytology to diagnose MTC. Patients at early stages were more prone to achieve $\mathrm{BC}$, which was a favorable prognostic factor. To the best of
\end{abstract}

Correspondence to: Dr Joana Simões-Pereira, Department of Endocrinology, Portuguese Institute of Oncology Francisco Gentil, Rua Professor Lima Basto, Lisbon 1099-023, Portugal

E-mail: joanasimoespereira@gmail.com

Key words: medullary thyroid carcinoma, calcitonin, RET, multiple endocrine neoplasia type 2 our knowledge, the present study reports for the first time that age at diagnosis is not a predictor of survival for patients with MTC when cases diagnosed by genetic/pentagastrin screening (who are usually young patients at the initial stages of the disease), are excluded from the analysis.

\section{Introduction}

Medullary thyroid carcinoma (MTC) accounts for 5-10\% of all thyroid cancer cases (1). MTC is a neuroendocrine tumor derived from parafollicular or ' $\mathrm{C}$ ' cells that secrete calcitonin (CT), which is a sensitive serum biomarker, useful for diagnosis of MTC and postoperative follow-up. MTC may be sporadic or familial, either in an isolated form [familial MTC (FMTC)] or as part of multiple endocrine neoplasia (MEN) type $2 \mathrm{~A}$ or $2 \mathrm{~B}$ syndromes (2). Familial forms of MTC are associated with mutations in the proto-oncogene rearranged during transfection (RET), which are transmitted by an autosomal dominant mode. Genetic testing enables an early diagnosis among relatives of patients with FMTC. By contrast, the diagnosis of familial index cases and sporadic tumors mainly relies on fine-needle aspiration cytology (FNAC), which has a considerable number of false-negative results that may delay the diagnosis (3). Measurement of serum CT levels in nodular thyroid diseases is not unanimously considered a routine procedure. Surgery is the most effective treatment for these tumors $(4,5)$.

The overall cause specific mortality of these tumors is $13.3-32.6 \%$ and $21.6-38.6 \%$ at 5 and 10 years, respectively (6). In previously reported cases, the main independent prognostic factors with influence on survival have been age and stage at diagnosis (survival rates at 10 years vary between $100 \%$ for patients in stage I and 20\% for those diagnosed at stage IV) (7).

The aim of the present study was to determine the demographic, clinical and pathological characteristics of MTC patients followed-up at the Portuguese Institute of Oncology Francisco Gentil (Lisbon, Portugal).

\section{Materials and methods}

The medical files of 140 patients with MTC who were diagnosed between 1990 and 2010 were reviewed in the present study. Cases were identified through the Portuguese South Regional Cancer Registry (Lisbon, Portugal) (http:// www.ror-sul.org.pt/) and from the database of the Department 
of Endocrinology of the Portuguese Institute of Oncology Francisco Gentil. This study was approved by the Ethics Committee of our center. Written informed consent was obtained from patients submitted to genetic testing. Inclusion criterion was histological diagnosis of MTC, which was reviewed by pathologists at the Portuguese Institute of Oncology Francisco Gentil in those cases of patients who had been operated at other institutions. Familial cases subjected to prophylactic surgery and reported by the pathologists as C-cell hyperplasia were not included in the study. Screening of familial cases was performed by $\mathrm{CT}$ stimulation with pentagastrin until 1994, and by RET mutation analysis thereafter. Calcitonin assay was performed by different methods over the years, from July 1988 to July 2000: ELSA-hCT (CIS bio international, Gif-sur-Yvette, France); solid-phase two-site immunoradiometric assay (IRMA), Reference Interval (RI) $<10 \mathrm{pg} / \mathrm{ml}$; from July 2000 to June 2006: IRMA hCT (CIS bio international, Gif-sur-Yvette, France); IRMA, RI $<10$ pg/ $\mathrm{ml}$; from June 2006 to present time: Calcitonin Immulite 2000 (Siemens Healthcare Diagnostics, Llanberis, Gwynedd, United Kingdom); IRMA, RI $<8.5$ pg/ml. Tumor stages were defined according to the tumor-node-metastasis (TNM) classification (8): Stage I (T1, N0 and M0); stage II (T2, N0 and M0); stage III (T3, N0 and M0 or T1-3, N1a and M0); stage IVA (T4a, any $\mathrm{N}$ and M0 or T1-T3, N1b and M0); stage IVB (T4b, any $\mathrm{N}$ and $\mathrm{M} 0$ ); and stage IVC (any T, any N and M1). Biochemical cure (BC) following surgery was considered when the CT levels measured at 3-6 months following surgery were lower than the reference values. Recurrence was defined as the reappearance of high levels of CT or locoregional and/ or distant metastasis following a period of no evidence of disease. Patients were classified based on their status at the last follow-up as succumbed to MTC, succumbed to other causes, alive without disease, alive with disease (biochemical or locoregional and/or distant metastasis) or not available for follow-up.

Statistical analysis was performed with SPSS version 20.0 (IBM SPSS, Armonk, NY, USA). $\chi^{2}$ test was used to compare categorical variables, while one-way analysis of variance and Tukey's test were used to compare means. Cumulative survival curves were constructed using the Kaplan-Meier method; log rank test was used to evaluate differences in the survival between groups and an adjusted Cox regression model was performed to find the independent prognostic factors with influence on survival. Age was assessed as a categorical variable ( $\leq 45$ versus $>45$ years) in this analysis. $\mathrm{P}<0.05$ was considered to indicate a statistically significant difference.

\section{Results}

Of the 140 patients enrolled in the study, 87 (62.1\%) were diagnosed with MTC at the Portuguese Institute of Oncology Francisco Gentil, whereas the remaining patients were referred to the above hospital following a first surgical intervention performed elsewhere. The average number of patients treated per year was 6.7. In total, 80 (57.1\%) patients were females (female:male ratio, 1.3:1.0). Patients with FMTC were significantly younger than patients with sporadic MTC $(33 \pm 20$ vs. $57 \pm 15$ years, respectively; $\mathrm{P}<0.05)$. Among the familial cases, there were $12 \mathrm{MEN}$ type $2 \mathrm{~A}, 1 \mathrm{MEN}$ type $2 \mathrm{~B}$
Table I. Histological characteristics of patients with medullary thyroid carcinoma.

\begin{tabular}{lr} 
Histological characteristics & Patient \\
\hline Tumor size, cm (mean \pm standard deviation) & $3.6 \pm 2.3$ \\
Side, \% & \\
Right lobe & 48 \\
Left lobe & 35 \\
Right and left lobes & 17 \\
Multifocality, \% & 23 \\
Angioinvasion, \% & 43 \\
Extrathyroidal extension, \% & 44 \\
Lymph node metastasis, \% & \\
Total & 43 \\
N1 & 5 \\
N1a & 5 \\
N1b & 90 \\
Associated histological diagnosis & \\
Total, \% & 33 \\
Follicular hyperplasia, $n$ & 19 \\
C-cell hyperplasia, $\mathrm{n}$ & 10 \\
Papillary carcinoma, $\mathrm{n}$ & 4 \\
Follicular carcinoma, $\mathrm{n}$ & 3 \\
Lymphocytic thyroiditis, $\mathrm{n}$ & 8
\end{tabular}

and 3 FMTC. Of these patients, 4 were index and 12 were diagnosed by genetic/pentagastrin screening across 7 different families. The genetic profiles and results of early thyroidectomy of the familial cases analyzed in the present study were previously reported by Bugalho et al (9).

The underlying conditions leading to the diagnosis of MTC in patients not detected by screening were: Presence of thyroid nodules in 105 cases; cervical lymph nodes in 12 cases; distant metastases in 2 cases; increased serum CT levels in 2 cases; increased serum carcinoembryonic antigen levels in 1 patient; incidental finding in 1 patient operated for a larynx carcinoma; and in 5 patients the reason was not specified in their clinical files. Neck symptoms were present in 23 cases, while 15 patients complained of diarrhea, 6 of which had distant metastases. A total of 14 patients exhibited distant metastases at diagnosis, 5 of them in the liver, 4 in the lungs, 2 in the bones, 2 in the liver, lungs and bones, and 1 in the liver and bones.

Among the 128 (91.4\%) patients not detected by screening, $44(34.4 \%)$ did not exhibit a preoperative suspicion of MTC, since FNAC was either negative for MTC or not requested and serum CT determination was not performed. FNAC and serum CT assay sensitivities were $51.3 \%$ and $98.7 \%$, respectively. Patients with a negative FNAC for MTC (58 cases) had cytological findings reported as non-diagnostic in 6 cases, benign in 13 cases and suspicious for a different tumor in 39 cases. In total, $129(92.1 \%)$ patients were subjected to surgery, and total thyroidectomy with cervical lymph node clearance was performed in 72 cases (level VI in 6 of them), while total thyroidectomy was performed in 5 patients and hemithyroidectomy in 6 patients. Of the remaining 11 patients, 7 underwent palliative surgery, 


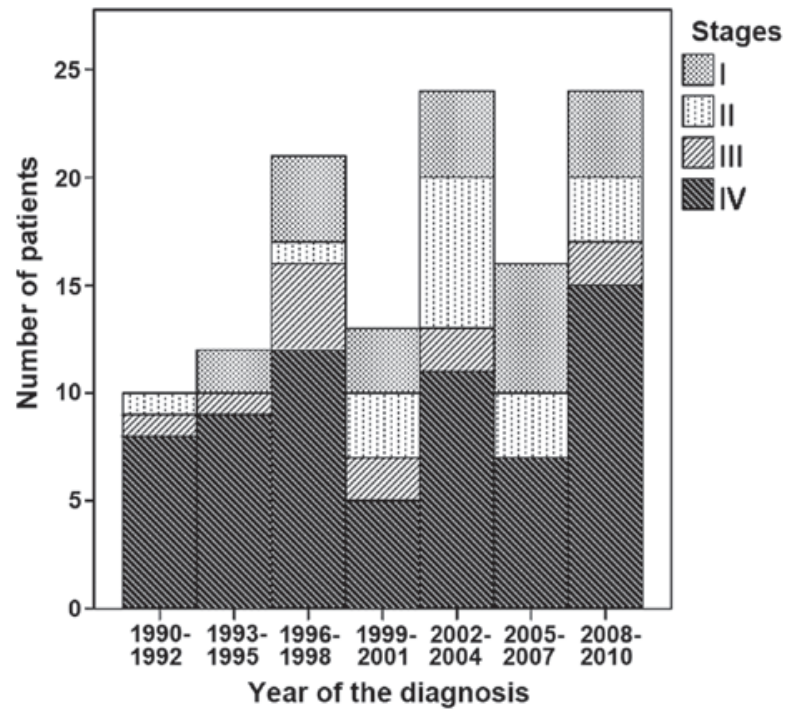

Figure 1. Stages at diagnosis of medullary thyroid carcinoma along the years.

while 4 were not operated due to advanced disease. Neck radiotherapy was performed in 21 (15.0\%) patients, with a palliative intention in 18 cases and in an adjuvant setting in 3 patients. Of the patients with advanced disease, 9 (6.4\%) were treated with classic chemotherapy and/or off-label kinase inhibitors. The histological characteristics of the patients are presented in Table I. Somatic mutations in the RET, Ras and B-Raf genes were evaluated in 53 patients, and the results were previously published by Moura et al $(10,11)$.

Staging at diagnosis was possible in 120 patients (Fig. 1), and demonstrated that $23(19.2 \%)$ patients were in stage I, $18(15.0 \%)$ in stage II, $12(10.0 \%)$ in stage III and $67(55.8 \%)$ in stage IV (of which, 42 were in stage IVA, 11 in stage IVB and 14 in stage IVC) $(\mathrm{P}<0.05)$. The majority of the sporadic cases [72 (68.6\%)] and all familial index cases presented with advanced stage (III-IV) whilst $72.7 \%$ (8 patients) of familial cases, detected by screening, were diagnosed at early stages of disease (stages I and II) ( $\mathrm{P}=0.008)$. Among 116 patients with serum CT evaluation following primary surgery, BC was achieved in 46 patients (39.7\%). Of these, only 3 patients $(6.5 \%)$ relapsed, 1 of them with biochemical recurrence and 2 with recurrence in the thyroidectomy surgical bed.

The median follow-up time was 5 years. At the last follow-up, 32 (22.9\%) patients had succumbed to MTC, while $4(2.9 \%)$ had deceased due to other causes, 38 (27.1\%) were in remission and 38 (27.1\%) were alive with disease. The remaining 28 patients $(20.0 \%)$ were not available for follow-up. In the group of patients that were alive with disease, biochemical evidence of disease (high serum CT levels) was present in $17(44.7 \%)$ cases, while structural disease (locorregional disease, metastatic cervical lymph nodes or a mass in thyroidectomy surgical bed) was detected in 15 patients (39.5\%) and distant metastasis in 6 patients (15.8\%).

Survival analysis. The survival rates at 5 and 10 years were 79.3 and $73.6 \%$, respectively, while median survival was not reached. When survival rate was analyzed for tumor stage at diagnosis, only 1 patient in stage II was observed to succumb to MTC, while no patients in stages I or III succumbed to MTC.
A

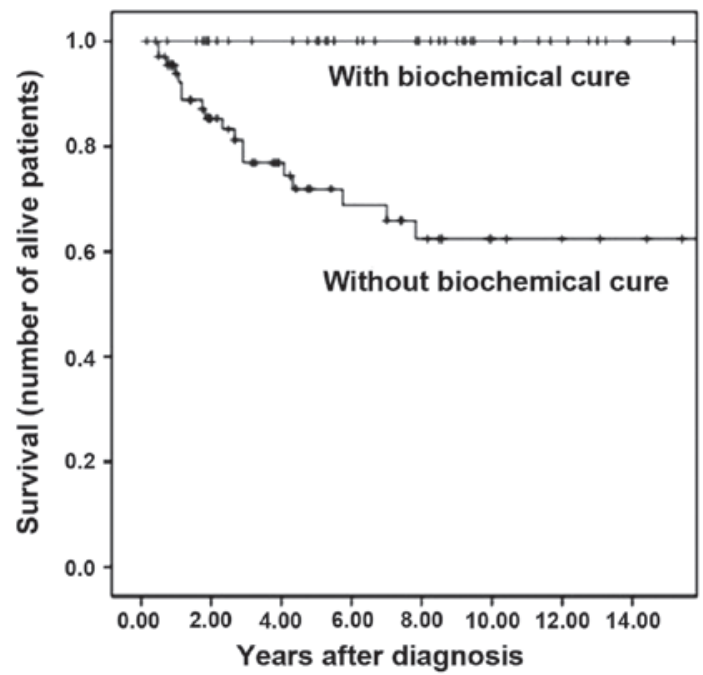

B

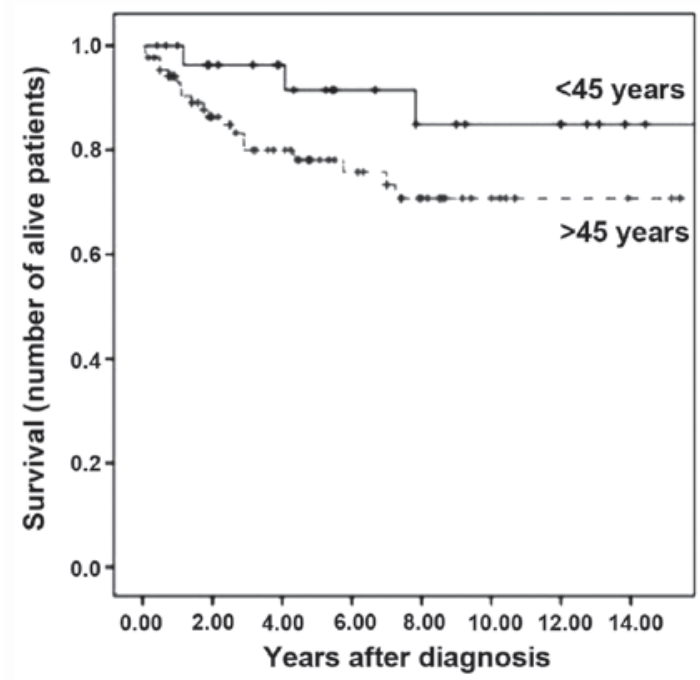

Figure 2. Univariate analysis. (A) Biochemical cure following primary surgery was a favorable prognostic factor $(\mathrm{P}<0.001)$. (B) Age did not influence survival when patients diagnosed by genetic screening or calcitonin stimulation with pentagastrin were excluded from the analysis $(\mathrm{P}=0.077)$.

The survival rates at 5 and 10 years for patients diagnosed with stage IV MTC were 58.6 and $49.0 \%$, respectively. The patient in stage II who succumbed to MTC had been subjected to total thyroidectomy plus unilateral cervical lymph node dissection. His initial TNM staging was T2NOM0, and a RET somatic mutation in codon 918 was also detected in the tumor. Biochemical evidence of disease persisted, and 8 months following surgery, liver and bone metastases were detected. The serum CT levels of the patient during his last follow-up were $97,706 \mathrm{pg} / \mathrm{ml}$. The patient succumbed to disease 4 months later. None of the patients who achieved BC succumbed to MTC, and the survival rates at 5 and 10 years for patients with postoperative hypercalcitoninemia were 71.9 and $62.4 \%$, respectively. Patients detected by genetic screening exhibited a $100.0 \%$ survival rate at 5 and 10 years, while sporadic MTC patients exhibited a survival rate at 5 and 10 years of 78.3 and $71.4 \%$, respectively. For familial index cases, the survival rate at 5 and 10 years was 75.0 and $37.5 \%$, respectively.

Univariate analysis was initially performed with the 129 patients who underwent surgery with a curative intent. The following adverse prognostic factors for survival were 
identified: Age $>45$ years $(\mathrm{P}=0.026)$, advanced stage at diagnosis $(\mathrm{P}=0.0001)$ and absence of $\mathrm{BC}(\mathrm{P}=0.00001)$. When patients detected by genetic/pentagastrin screening were excluded from the analysis, age was no longer a prognostic factor $(\mathrm{P}=0.077)$, but stage of disease remained a strong predictor of survival $(\mathrm{P}<0.001)$ (Fig. 2). Multivariate analysis, performed with the initial 129 patients, revealed that only the absence of $\mathrm{BC}(\mathrm{P}=0.031 ; 95 \%$ confidence interval, 0.001-0.691) had a negative impact on prognosis.

\section{Discussion}

The incidence of MTC among the present patients remained stable throughout the years, although a high proportion of advanced cases was observed, probably due to the absence of routine serum CT determination in the management of thyroid nodules. At diagnosis, MTC was frequently revealed in an advanced stage of disease, as observed by other authors $(6,12)$. A diagnosis of MTC, either in sporadic or in familial index cases, is usually suspected by FNAC and/or by detection of high serum CT levels in patients with thyroid nodules. In the present study, the sensitivities of FNAC and serum CT analysis for the diagnosis of MCT were 51.3 and $98.7 \%$, respectively. Among the patients analyzed in the present study, there was only 1 patient whose levels of preoperative CT (determined at another institution) were below the reference value. Following total thyroidectomy, the CT levels of this patient (measured at the Portuguese Institute of Oncology Francisco Gentil) were elevated, thus ruling out the possibility of a non-secreting tumor. Routine CT determination in thyroid nodules is a controversial issue $(2,13)$. For instance, Elisei et al (3), performed CT determination in 10,864 patients with thyroid nodules, and detected 1 case of MTC in every 250 patients screened. The authors verified that CT determination enabled a diagnosis of MTC at an early stage with 10-year survival rates higher than those observed in MCT cases who were not detected by screening. In the present study, in $67.2 \%(39 / 58)$ of patients with a negative FNAC, the cytological findings were suspicious for carcinoma. Thus, FNAC provided an indication for surgery in $\sim 2 / 3$ of patients with negative-MTC FNAC. However, in the present cohort, $22 \%$ of the patients would have not been subjected surgery due to a benign FNAC result. This value was higher than that previously published by the present authors in a smaller cohort (14), which was similar to the value described by Elisei et al (3), but lower than that reported by Papaparaskeva et al (15), whereby FNAC was positive for MTC in $89.0 \%(81 / 91)$ of the cases analyzed.

Of the 140 patients included in the present study, only $11 \%$ represented familial cases, which indicates a prevalence significantly lower than that reported by others $(6,16-19)$. However, the present study only included patients with MTC confirmed by histology, which may have underestimated the total number of familial cases. Patients detected by genetic screening were diagnosed at younger ages than sporadic cases, which is in agreement with the literature $(6,19,20)$, and were also diagnosed at earlier stages of the disease $(6,19,20)$, presenting 5 and 10-year survival rates of $100.0 \%$. This underlines the importance of early screening for mutation carriers. It also supports the idea that germline mutations should be investigated in sporadic cases, particularly in those with multifocality or C-cell hyperplasia, since $\leq 10 \%$ of these patients represent hereditary MTC index cases $(9,12)$. In agreement with previous studies $(21,22)$, mutation in the codon 634 in the RET gene was the most prevalent among the present cohort. In previous studies published by the present authors $(10,11)$, it was demonstrated that patients with 918 and 883 somatic mutations were associated with aggressive disease and higher number of lymph nodes metastases, higher percentage of multifocality, persistent hypercalcitoninemia following surgery and more advanced stages of disease at presentation than those with other RET mutations. Patients with Ras mutations exhibited an intermediate behavior between these two groups. Therefore, these results suggest that analyzing tumor DNA for RET and Ras mutations may be important, since their impact in patient prognosis may differ.

Specific survival at 5 and 10 years in the present cohort was 79.3 and $73.6 \%$, respectively, which was similar to previous reports $(16,19,20,22,23)$. Another possible adverse prognostic factor identified in the present study was age $>45$ years. However, when patients detected by genetic/pentagastrin screening were omitted in the univariate analysis, age was no longer a prognostic factor for survival, contrarily to disease stage, which remained a significant factor. This is due to the fact that patients detected by genetic screening were significantly younger than index or sporadic cases and exhibited a survival rate at 5 and 10 years of $100.0 \%$. Therefore, the influence of age on prognosis should be carefully assessed in cohorts with a high number of hereditary MTC cases, such as those available in the literature $(4,6)$. This is important considering that there are certain studies (6) who defend that age should be considered in the staging of MTCs, as it happens in thyroid cancer of the follicular epithelium.

In the present study, postoperative CT was the only prognostic factor observed to exert a significant impact on survival, as previously described by other authors (20). In fact, of the $40.0 \%$ of patients who achieved BC following the first surgical procedure, only 3 patients $(6.5 \%)$ relapsed. Therefore, complete resection should be performed, if feasible.

The present cohort consisted of a considerable number of MTC patients followed-up in a single institution (and therefore, with uniform criteria for collecting data) and with a median follow-up time similar to other published studies $(6,16)$. However, the current study presents certain limitations: i) It is a retrospective analysis that included patients referred from other institutions; thus, it was not possible to retrieve certain information for these patients. In addition, the surgical protocols for these patients may have varied across institutions, which may have influenced the results in terms of cure, survival and recurrence; ii) since the studied period was long, it is likely that the surgical protocols varied for each tumor stage over the years.

In conclusion, the present study confirms that the majority of MTCs are detected in advanced stages of disease, and that CT determination is more sensitive than FNAC in the diagnosis of these types of carcinoma. Furthermore, the present study demonstrated that the annual incidence of these tumors has remained stable for the past two decades, contrarily to the 'epidemic' increase that has been observed for papillary thyroid carcinomas. Once subjected to primary surgery treatment, if 
$\mathrm{BC}$ is achieved, relapse of MTC is rarely observed. To the best of our knowledge, the present study is the first to report that age is only capable of acting as a potential prognostic factor if patients detected by genetic screening are considered in the analysis, since these patients are usually diagnosed earlier and at initial stages of the disease.

\section{References}

1. Hundahl SA, Fleming ID, Fremgen AM and Menck HR: A National Cancer Data Base report on 53,856 cases of thyroid carcinoma treated in the U.S., 1985-1995. Cancer 83: 2638-2648, 1998.

2. Kloos RT, Eng C, Evans DB, Francis GL, Gagel RF, Gharib H, Moley JF, Pacini F, Ringel MD, Schlumberger $M$ and Wells SA Jr; American Thyroid Association Guidelines Task Force: Medullary thyroid cancer: Management guidelines of the American Thyroid Association. Thyroid 19: 565-612, 2009.

3. Elisei R, Bottici V, Luchetti F, Di Coscio G, Romei C, Grasso L, Miccoli P, Iacconi P, Basolo F, Pinchera A and Pacini F: Impact of routine measurement of serum calcitonin on the diagnosis and outcome of medullary thyroid cancer: Experience in 10,864 patients with nodular thyroid disorders. J Clin Endocrinol Metab 89: 163-168, 2004.

4. Evans DB, Fleming JB, Lee JE, Cote G and Gagel RF: The surgical treatment of medullary thyroid carcinoma. Semin Surg Oncol 16: 50-63, 1999.

5. Fleming JB, Lee JE, Bouvet M, Schultz PN, Sherman SI, Sellin RV, Friend KE, Burgess MA, Cote GJ, Gagel RF and Evans DB: Surgical strategy for the treatment of medullary thyroid carcinoma. Ann Surg 230: 697-707, 1999.

6. Kebebew E, Ituarte PH, Siperstein AE, Duh QY and Clark OH: Medullary thyroid carcinoma: Clinical characteristics, treatment, prognostic factors, and a comparison of staging systems. Cancer 88: 1139-1148, 2000.

7. Leboulleux S, Baudin E, Travagli JP and Schlumberger M: Medullary thyroid carcinoma. Clin Endocrinol (Oxf) 61: 299-310, 2004

8. Greene FL, Page DL, Fleming ID, Fritz A and Balch DM: AJCC Cancer Staging Manual. 6th edition. Springer Verlag, Chicago, 2003.

9. Bugalho MJ, Domingues R, Santos JR, Catarino AL and Sobrinho L: Mutation analysis of the RET proto-oncogene and early thyroidectomy: Results of a Portuguese cancer centre. Surgery 141: 90-95, 2007

10. Moura MM, Cavaco BM, Pinto AE, Domingues R, Santos JR, Cid MO, Bugalho MJ and Leite V: Correlation of RET somatic mutations with clinicopathological features in sporadic medullary thyroid carcinomas. Br J Cancer 100: 1777-1783, 2009.

11. Moura MM, Cavaco BM, Pinto AE and Leite V: High prevalence of RAS mutations in RET-negative sporadic medullary thyroid carcinomas. J Clin Endocrinol Metab 96: E863-E868, 2011.
12. Marsh DJ, Learoyd DL and Robinson BG: Medullary thyroid carcinoma: Recent advances and management update. Thyroid 5: 407-424, 1995.

13. Gharib H, Papini E, Paschke R, Duick DS, Valcavi R, Hegedüs L and Vitti P; AACE/AME/ETA Task Force on Thyroid Nodules: American Association of Clinical Endocrinologists, Associazione Medici Endocrinologi, and European Thyroid Association medical guidelines for clinical practice for the diagnosis and management of thyroid nodules: Executive summary of recommendations. J Endocrinol Invest 33 (Suppl 5): S51-S56, 2010.

14. Bugalho MJ, Santos JR and Sobrinho L: Preoperative diagnosis of medullary thyroid carcinoma: Fine needle aspiration cytology as compared with serum calcitonin measurement. J Surg Oncol 91: 56-60, 2005.

15. Papaparaskeva K, Nagel H and Droese M: Cytologic diagnosis of medullary carcinoma of the thyroid gland. Diagn Cytopathol 22: $351-358,2000$

16. Cupisti K, Wolf A, Raffel A, Schott M, Miersch D, Yang Q, Eisenberger CF, Röher HD and Knoefel WT: Long-term clinical and biochemical follow-up in medullary thyroid carcinoma: A single institution's experience over 20 years. Ann Surg 246: 815-821, 2007.

17. Wells SA Jr and Franz C: Medullary carcinoma of the thyroid gland. World J Surg 24: 952-956, 2000.

18. Kameyama $\mathrm{K}$ and Takami H: Medullary thyroid carcinoma: Nationwide Japanese survey of 634 cases in 1996 and 271 cases in 2002. Endocr J 51: 453-456, 2004.

19. Modigliani E, Cohen R, Campos JM, Conte-Devolx B, Maes B, Boneu A, Schlumberger M, Bigorgne JC, Dumontier P, Leclerc L, et al: Prognostic factors for survival and for biochemical cure in medullary thyroid carcinoma: Results in 899 patients. The GETC Study Group. Groupe d'etude des tumeurs à calcitonine. Clin Endocrinol (Oxf) 48: 265-273, 1998.

20. Grozinsky-Glasberg S, Benbassat CA, Tsvetov G, Feinmesser R, Peretz H, Shimon I and Lapidot M: Medullary thyroid cancer: A retrospective analysis of a cohort treated at a single tertiary care center between 1970 and 2005. Thyroid 17: 549-556, 2007.

21. Rohmer V, Vidal-Trecan G, Bourdelot A, Niccoli P, Murat A, Wemeau JL, Borson-Chazot F, Schvartz C, Tabarin A, Chabre O, et al; Groupe Français des Tumeurs Endocrines: Prognostic factors of disease-free survival after thyroidectomy in 170 young patients with a RET germline mutation: A multicenter study of the Groupe Français d'Etude des Tumeurs Endocrines. J Clin Endocrinol Metab 96: E509-E518, 2011.

22. Frank-Raue K, Buhr H, Dralle H, Klar E, Senninger N, Weber T, Rondot S, Höppner W and Raue F: Long-term outcome in 46 gene carriers of hereditary medullary thyroid carcinoma after prophylactic thyroidectomy: Impact of individual RET genotype. Eur J Endocrinol 155: 229-236, 2006.

23. Dottorini ME, Assi A, Sironi M, Sangalli G, Spreafico G and Colombo L: Multivariate analysis of patients with medullary thyroid carcinoma. Prognostic significance and impact on treatment of clinical and pathologic variables. Cancer 77: $1556-1565,1996$. 